\title{
Common-Mode Insertion Indices Compensation With Capacitor Voltages Feedforward to Suppress Circulating Current of MMCs
}

\author{
Xiaoling Xiong, Xiongfei Wang, Dong Liu, Frede Blaabjerg, and Chengyong Zhao
}

\begin{abstract}
In order to improve the efficiency of modular multilevel converters (MMCs) and reduce the capacitor voltage ripples, the circulating current between the phase-legs should be suppressed. In existing circulating current suppression control (CCSC) methods, multiple resonant regulators or $d q$-frame integrators are usually employed to reduce the harmonic components. Besides the complexity, it requires additional designs of controller parameters, which relies on accurate frequency and sequence information of the circulating currents. In this paper, a common-mode $(\mathrm{CM})$ insertion indices compensation method is introduced to realize CCSC, which is based on feeding forward the capacitor voltages. This approach not only facilitates the analysis of circulating current mechanism with a deep insight, but also provides a simple approach to achieve a wide bandwidth circulating current suppression without considering the specific harmonic frequencies and sequences. The latter one makes it easy to be used even in the unbalanced, multi-frequency or variable fundamental frequency ac systems. In addition to these advantages, the proposed compensation method can maintain inter-arm capacitor voltages balanced without additional differential energy control loop, which is required in the existing capacitor voltage feedforward scheme. Analytical and extensive simulation results are given for validating the effectiveness of the proposed approach.
\end{abstract}

Index Terms-Capacitor voltages feedforward, circulating current suppression control, common-mode insertion indices, compensation method, modular multilevel converter (MMC).

\section{INTRODUCTION}

NINCE the modular multilevel converter (MMC) was inStroduced in 2001 by Professor R. Marquardt for high voltage direct (HVDC) transmission [1], it is gaining global

Manuscript received January 13, 2020; revised May 27, 2020; accepted May 27, 2020. Date of publication June 30, 2020; date of current version June 18, 2020. This work was supported by the National Natural Science Foundation of China under Grant 51707065. (Corresponding author: Xiaoling Xiong)

X. Xiong and C. Zhao are with the State Key Laboratory of Alternate Electrical Power System with Renewable Energy Sources, North China Electric Power University, Beijing, China (e-mail: xiongx11102@ncepu.edu.cn; chengyongzhao@ncepu.edu.cn).

X. Wang and F. Blaabjerg are with the Department of Energy Technology, Aalborg University, Aalborg 9220, Denmark (e-mail: xwa@et.aau.dk; fbl@ et.aau.dk).

D. Liu is with the HVDC Technology Department, State Grid Corporation of China, Beijing, China (e-mail: 16039662@qq.com).

Digital Object Identifier 10.24295/CPSSTPEA.2020.00009 growing attentions [2], [3]. Due to its excellent features, e.g. high modularity and voltage scalability, independent active power and reactive power control, low losses and low switching frequency, etc. [4], the MMC has become one of the most promising multilevel converter topology and has been extended to many medium/high power applications [2], [3], [5].

In contrast to conventional two-level converters, the major different feature of MMCs is the the circulating current that flows between the phase-legs, which increases the root-mean-square (RMS) value of the arm currents significantly [6], consequently increasing the losses and power ratings of the devices.

Undesirable circulating currents are internal currents that flow among MMC legs but do not flow outside, including the loworder harmonic currents and switching frequency components. The latter one is caused by instantaneous changes among voltage levels at switching frequency, which is due to multilevel modulation. In this stage, the modified carrier disposition modulation [7] or shifting the phases of arm SM PWM carriers [8] can be used to minimize the switching ripples. It can also be limited by the presence of a large number of SMs in one arm with the nearest level control method. [9]. In contrast, the low-order harmonic circulating currents are caused by SM capacitor voltage ripples coupled into the $\mathrm{CM}$ voltage via the direct modulation method, and this can not be minimized by the primary PWM process or by increasing the number of SMs. Although these low-order circulating currents can be reduced to some extent by larger SM capacitors and larger arm inductors, it is costly. Commonly, it is expected that the low-order circulating currents should be eliminated or at least suppressed to a small magnitude with a control technique, in order to reduce the losses and stresses of semiconduct devices [9].

Many efforts have been made to suppress the low-order harmonic circulating currents, which can generally be classified into two types [10], one is the direct method, e.g. the conventional CCSC method, and the other is an indirect compensation method by the capacitor-voltage feedforward control. The direct suppression method assumes that the frequencies and sequences of circulating current components are already known, which only contain even order harmonics in steady state, symmetric grid conditions, and the negativesequence 2nd-order harmonics are the dominant component for a three-phase MMC [11], [12]. Thus, the major circulating currents are eliminated by employing a negative-sequence 
double-line-frequency $d q$ transformation with a pair of integral regulators in the rotating $d q$ frame [13], [14]. One main problem associated with this method is the robustness under the ac side imbalances, where a large positive-sequence 2nd-order component exists. Therefore, the above method with double line-frequency $d q$ transformation requires a complicated sequence extraction procedure [15], [16]. It is also inconvenient to be implemented in the non-three-phase system, such as the virtual circuit needs to be used in a twophase system [17]. Another problem with this method is that other frequency harmonic currents are not suppressed, which becomes more serious when optimized control methods are adopted. For example, the zero-sequence 3rd-order voltage injection [18], [19] is used to extend the modulation range and reduce capacitor voltage ripples, but the 4th-order harmonics in the circulating current is becoming larger. Moreover, as this method is highly dependent on the ac system frequency and sequence, which can be further affected by the phase locked loop used to detect the phase of the grid voltage. To overcome these limitations, multiple resonant regulators can be used in a stationary $\alpha \beta$ frame [20]-[22], each of them is designed to suppress one frequency harmonic component. Model predictive control are also proposed to suppress the circulating currents [23], but the computational complexity drastically increases with large number of SMs. Alternatively, the repetitive control is employed [24] to suppress all even harmonics with a simpler parameter design. However, all these methodologies require the frequency and sequence information of the circulating currents, additional resonant or integrated controllers are needed to be designed.

On the other hand, the indirect control method in [25] proposed a closed-loop modulation by feed-forwarding the instantaneous arm capacitor voltages independently to compensate the upper and lower arm insertion indices. However, the internal dynamics with this method will be marginally stable as the average capacitor voltages get out of control [26], two additional energy control loops are inevitably required. To solve this problem, an open-loop control method has been proposed where the estimated capacitor voltages were used to replace the measured ones [27], which has been demonstrated to be able to achieve global asymptotic stability [28], [29]. However, explicit expressions [27] are not always practical for the online implementation in a control system, several trigonometric and nonlinear functions need to be calculated online. Furthermore, the performance of this method is highly dependent on the accuracy of system parameters. To get the capacitor voltage, the online band-pass filter is employed to replace integral of the capacitor current in [29]. This method is highly dependent on the grid frequency, and becomes more complicated or even infeasible when the ac system frequency varies. Moreover, it is inaccurate during the transient period as the calculations and analysis are based on the steady state. Alternatively, one compensation method was proposed in [30], which forward predicted the SM dc-link voltage to suppress the circulating currents. However, this prediction method is generated at each half-carrier switching interval that is based on

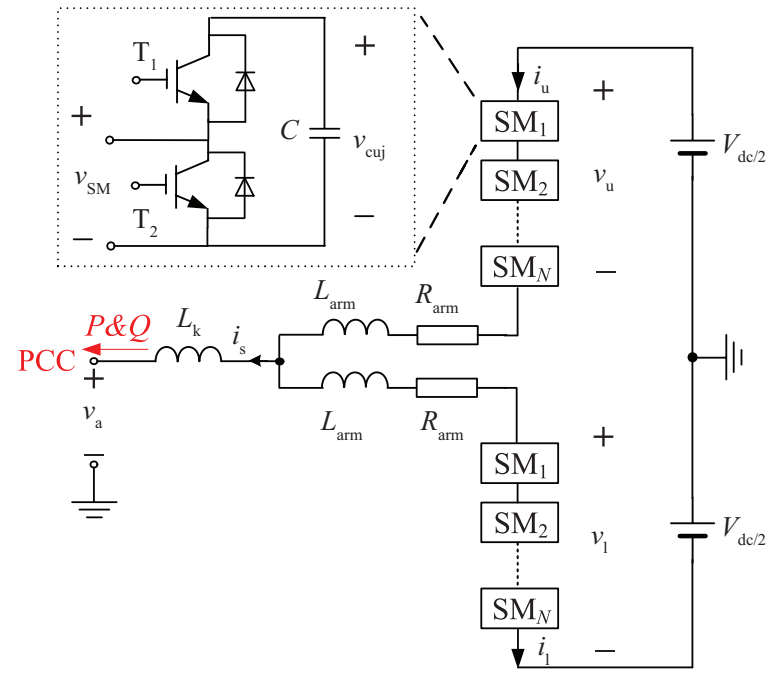

Fig. 1. Single phase diagram of an MMC and its submodule topology.

the PWM schemes, it can not be used in the NLC modulation with the sorting balance algorithms.

The paper is organized as follows. Section II describes the commonly used averaged model of the MMC, which is used to analyze the mechanism of the circulating currents in detail. According to this analysis, a new CM insertion indices compensation algorithm is derived and implemented to suppress the circulating currents in Section III. In Section IV, the voltage balance capability of the inter-arm capacitors with different CCSC methods is analyzed and compared. A simulation model based on a three-phase MMC is established in Section V, simulation results are presented to validate the analysis of circulating current and the developed CCSC strategy. Section VI concludes this paper.

\section{Circulating CuRRENT OF AN MMC}

Since the MMC is three-phase symmetric, only one phase is analyzed as an example. Fig. 1 shows a single phase diagram of an MMC, which consists of two arms (upper arm and lower arm), each made up by $N$ SMs connected in series with an arm inductor $L_{\text {arm }}$. In this work, each SM is a typical half bridge, which is composed by two IGBTs $\left(\mathrm{T}_{1}\right.$ and $\mathrm{T}_{2}$ ) and a storage capacitor $C$. The output voltage $v_{\mathrm{SM}}$ is equal to the capacitor voltage $v_{\text {cuj }}$ when the SM is inserted or zero when the $\mathrm{SM}$ is bypassed. $L_{\mathrm{k}}$ is the equivalent leakage inductance of the connected transformer plus line inductance. $R_{\text {arm }}$ is the equivalent resistance, which represents the losses of the relative arm. $v_{\mathrm{u}}, v_{1}, i_{\mathrm{u}}$ and $i_{1}$ are the upper and lower arm voltages and arm currents, respectively. $v_{\mathrm{a}}$ and $i_{\mathrm{s}}$ are the output ac voltage and current at the point of common coupling (PCC), respectively. $V_{\mathrm{dc}}$ is the DC side voltage, which is split into two DC sources connected in series and its central point is grounded to the ac side ground.

\section{A. Averaged Model of MMCs}

By controlling the insertion indices $n_{\mathrm{u}}$ and $n_{\mathrm{l}}$, the inserted number of SMs varies between 0 and $N$, accordingly, the 


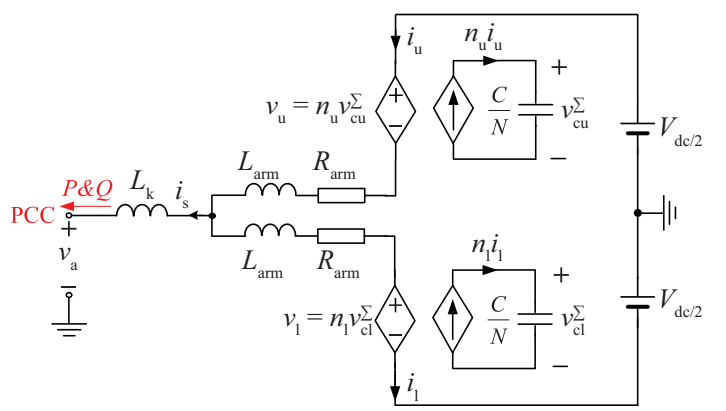

(a)

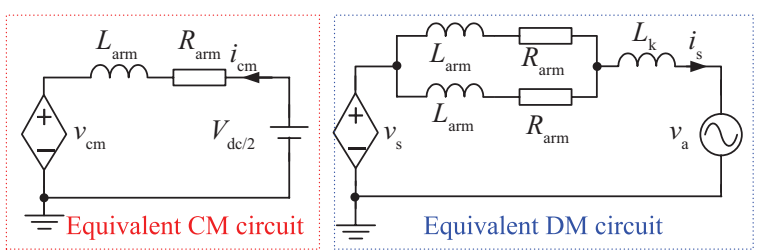

(b)

Fig. 2. (a) Commonly used averaged model for one phase of MMC. (b) The equivalent common-mode and differential-mode circuit.

arm voltage $v_{\mathrm{u}}$ and $v_{1}$ can be varied as desired between 0 and the sum of capacitor voltages $v_{\mathrm{cu}}^{\Sigma}, v_{\mathrm{cl}}^{\Sigma}$. Assuming that $N$ is large enough to allow the switching harmonics smoothed and can be neglected, so that $v_{\mathrm{u}}, v_{1}$ and $n_{\mathrm{u}}, n_{1}$ can be treated as continuous variables. Then the averaged model [28], [29] can be derived, as shown in Fig. 2(a), each arm includes an equivalent capacitor $C / N$.

From Fig. 2(a), the differential-mode (DM) current $i_{\mathrm{s}}$ can be obtained using Kirchhoff's current law, which is $i_{\mathrm{s}}=i_{\mathrm{u}}-i_{1}$. Defining that $i_{\mathrm{cm}}$ represents the CM current that is comprised of $i_{\mathrm{cm} 0}$, the dc component, and $i_{\text {cir }}$, which is the circulating currents induced by the coupling effects with the direct modulation. Thus $i_{\mathrm{cm}}$ can be expressed as

$$
i_{\mathrm{cm}}=\frac{i_{\mathrm{u}}+i_{1}}{2}=i_{\mathrm{cm} 0}+i_{\mathrm{cir}}
$$

meanwhile, the CM voltage $v_{\mathrm{cm}}$ and the DM voltage $v_{\mathrm{s}}$ are defined as

$$
\begin{aligned}
& v_{\mathrm{cm}}=\frac{v_{1}+v_{\mathrm{u}}}{2}=\frac{n_{1} v_{\mathrm{cl}}^{\Sigma}+n_{\mathrm{u}} v_{\mathrm{cu}}^{\Sigma}}{2} \\
& v_{\mathrm{s}}=\frac{v_{1}-v_{\mathrm{u}}}{2}=\frac{n_{1} v_{\mathrm{cl}}^{\Sigma}-n_{\mathrm{u}} v_{\mathrm{cu}}^{\Sigma}}{2}
\end{aligned}
$$

Applying Kirchhoff's voltage law to the upper and lower arm loops, we can analytically get the current dynamic relations as

$$
\begin{aligned}
& v_{\mathrm{s}}-R_{\mathrm{dm}} i_{\mathrm{s}}-L_{\mathrm{dm}} \frac{\mathrm{d} i_{\mathrm{s}}}{\mathrm{d} t}=v_{\mathrm{a}} \\
& v_{\mathrm{cm}}+R_{\mathrm{cm}} i_{\mathrm{cm}}+L_{\mathrm{cm}} \frac{\mathrm{d} i_{\mathrm{cm}}}{\mathrm{d} t}=\frac{V_{\mathrm{dc}}}{2}
\end{aligned}
$$

where $R_{\mathrm{dm}}=R_{\mathrm{arm}} / 2, L_{\mathrm{dm}}=L_{\mathrm{arm}} / 2+L_{\mathrm{k}}$ are equivalent resistance and inductance for the equivalent DM circuit; $R_{\mathrm{cm}}=R_{\mathrm{arm}}, L_{\mathrm{cm}}=$ $L_{\text {arm }}$ are equivalent resistance and inductance for the equivalent CM circuit, respectively.

Accordingly, the equivalent DM and CM circuits can be drawn in Fig. 2(b). It is obvious that $v_{\mathrm{s}}$ drives $i_{\mathrm{s}}$, and the voltage difference between $v_{\mathrm{a}}$ and $v_{\mathrm{s}}$ determines $i_{\mathrm{s}}$, crossing the RL impedance in the DM circuit. Meanwhile, $i_{\mathrm{cm}}$ is driven by the voltage difference between $V_{\mathrm{dc}} / 2$ and $v_{\mathrm{cm}}$, through the CM impedance. Thus, if $i_{\mathrm{cm}}$ is designed to be a pure dc current $i_{\mathrm{cm} 0}$, the CM voltage should be controlled such that $v_{\mathrm{cm}}=V_{\mathrm{dc}} / 2-$ $R_{\mathrm{arm}} i_{\mathrm{cm} 0} \approx V_{\mathrm{dc}} / 2$ (assuming $R_{\mathrm{arm}}$ is small enough), which is the designed ideal CM voltage. From Fig. 2, the capacitor dynamics can also be obtained as

$$
\begin{aligned}
& \frac{C}{N} \frac{\mathrm{d} v_{\mathrm{cu}}^{\Sigma}}{\mathrm{d} t}=n_{\mathrm{u}} i_{\mathrm{u}}=n_{\mathrm{u}}\left(\frac{i_{\mathrm{s}}}{2}+i_{\mathrm{cm}}\right) \\
& \frac{C}{N} \frac{\mathrm{d} v_{\mathrm{cl}}^{\Sigma}}{\mathrm{d} t}=n_{1} i_{1}=n_{1}\left(-\frac{i_{\mathrm{s}}}{2}+i_{\mathrm{cm}}\right)
\end{aligned}
$$

\section{B. The Mechanism of Circulating Currents Between Phase- Legs of an $M M C$}

For the conventional direct modulation, the insertion indices are given as:

$$
n_{\mathrm{u}}=\frac{\nu_{\mathrm{cm}}^{*}-v_{\mathrm{s}}^{*}}{V_{\mathrm{dc}}} \quad n_{\mathrm{l}}=\frac{\nu_{\mathrm{cm}}^{*}+v_{\mathrm{s}}^{*}}{V_{\mathrm{dc}}}
$$

where $v_{\mathrm{s}}^{*}$ and $v_{\mathrm{cm}}^{*}$ are the output of the DM and CM voltage references, respectively.

Without loss of generality, assuming that the CM and DM voltage reference are both equal to the ideal values for the direct voltage modulation strategy without CCSC, such as $v_{\mathrm{cm}}^{*}$ $=V_{\mathrm{dc}} / 2, v_{\mathrm{s}}^{*}=V_{\mathrm{s}} \cos \left(\omega_{1} t\right)$, thus $n_{\mathrm{cm}}=0.5$ and $n_{\mathrm{dm}}=0.5 \mathrm{~m} \cos \left(\omega_{1} t\right)$, where $m=2 V_{\mathrm{s}} / V_{\mathrm{dc}}$ is the modulation index. Consequently, $i_{\mathrm{cm}}$ and $v_{\mathrm{cm}}$ will contain the 2 nd order harmonics, $v_{\mathrm{s}}$ and $i_{\mathrm{s}}$ can be assumed to be pure sinusoidal for simplicity.

The voltage and current quantities in an MMC with the direct modulation strategy are circularly coupled by the insertion indices through (2), (3) and (4), which are also clearly shown in Fig. 3, where the upper arm is taking as an example. Noting that, only the low-order frequency components are considered. As shown in Fig. 3, the capacitor current for the two arms can be obtained as $i_{\text {capu }}=n_{\mathrm{u}} i_{\mathrm{u}}$ and $i_{\text {capl }}=n_{\mathrm{l}} i_{\mathrm{l}}$.

Then, integrating the capacitor current to get the capacitor voltage, yields

$$
\begin{gathered}
v_{\mathrm{cu}}^{\Sigma}=V_{\mathrm{c} 0}+\frac{N}{C \omega_{1}}\left(\Delta V_{\omega_{1}}+\Delta V_{\omega_{2}}+\Delta V_{\omega_{3}}\right) \\
v_{\mathrm{cl}}^{\Sigma}=V_{\mathrm{c} 0}+\frac{N}{C \omega_{1}}\left(-\Delta V_{\omega_{1}}+\Delta V_{\omega_{2}}-\Delta V_{\omega_{3}}\right)
\end{gathered}
$$

where $\Delta V_{\omega 1}, \Delta V_{\omega 2}$ and $\Delta V_{\omega 3}$ are the 1st, 2nd and 3rd-order ac 




Fig. 3. The relationships of electrical variables in the upper arm.

components in the capacitor voltage, respectively. The detailed analytical expressions is ignored here. This indicates that both arm capacitor voltages contain harmonic components from dc up to the 3rd-order due to the frequency coupling effects, and the odd frequency components of the upper and lower arm are opposite in phase whereas the even frequency components are in phase. The arm voltage can be derived by multiplying the capacitor voltage with the insertion indices. Again, the frequency coupling will induce the harmonics components to the arm voltages, and it will contain dc to 4th-order harmonics. The even components that are in phase for the upper and lower arms drive the circulating currents as shown in Fig. 2(b), where $i_{\text {cir }}$ contains the 2 nd and 4 th-order components, which are different from the precondition (we assumed it only had the 2nd-order harmonic firstly). What's worse, it will be again circularly coupled with the insertion indices as shown in Fig. 3, and more additional harmonics will be induced in the converter. The even frequency voltages of the two arms are in phase, forming the CM voltage $v_{\mathrm{cm}}$, which drives $i_{\mathrm{cm}}$, and consequently, $i_{\text {cir }}$ contains more even frequency components. The existing conventional CCSC method commonly adopts a resonant regulator or a $d q$ frame+PI regulator to suppress the major 2nd-order component, which, however can only eliminate one frequency harmonic. More regulators are needed to suppress more even harmonics, which is sufficiently complicated. Moreover, this method highly relies on the ac system frequency and sequence, which can be further affected by the dynamics of the phase locked loop.

From the above analysis, it can be concluded that the classical direct modulation is easy to be implemented but the inherent frequency coupling characteristics induce many harmonic components into the system, especially the even harmonics drive the circulating currents between the phase-legs. The frequency coupling effect is due to the capacitor voltage ripples are coupled into the $\mathrm{CM}$ voltage, which is not strictly equal to the designed ideal value $V_{\mathrm{dc}} / 2$ but contains harmonic components.

Moreover, the circulating currents and the corresponding common-mode voltages will contribute more components in the one-phase leg energy storage requirement, which will be eliminated or reduced when the circulating currents was suppressed. Due to the energy storage is related to the capacitor voltage, and $V_{\mathrm{dc}}$ is fixed, then the lower energy storage requirement means the lower capacitor voltage ripple, which can reduce the voltage stress of the devices.

\section{Proposed CCSC Compensation Strategy}

\section{A. Compensated Terms Derivation for Common-Mode Insertion Indices}

In this section, a CCSC method is proposed, which forces the $\mathrm{CM}$ voltage to achieve the designed value no matter what the capacitor voltages are. Then all additional harmonics in $\mathrm{CM}$ current can be eliminated regardless of the frequency and sequence information.

Assuming that $\Delta v_{\mathrm{cm}}$ is the additional compensation term, the insertion indices for the upper and lower arms in (5) after being compensated are changed as

$$
\begin{gathered}
n_{\mathrm{u}}^{\mathrm{c}}=\frac{v_{\mathrm{cm}}^{*}+\Delta v_{\mathrm{cm}}-v_{\mathrm{s}}^{*}}{V_{\mathrm{dc}}} \\
n_{\mathrm{l}}^{\mathrm{c}}=\frac{v_{\mathrm{cm}}^{*}+\Delta v_{\mathrm{cm}}+v_{\mathrm{s}}^{*}}{V_{\mathrm{dc}}}
\end{gathered}
$$

From (7), it can be seen that the sign of the compensated terms for the upper and lower arms are the same as they are designed to compensate the CM insertion indices. Only in this way, through (2), it can generate additional even components in the CM voltage $v_{\mathrm{cm}}$, which is then used to cancel the original even harmonic voltages in $v_{\mathrm{cm}}$. The compensated $\mathrm{CM}$ voltage of $\mathrm{MMC}$ is derived as

$$
v_{\mathrm{cm}}^{\mathrm{c}}=\frac{n_{1} v_{\mathrm{cl}}^{\Sigma}+n_{\mathrm{u}}^{\mathrm{c}} v_{\mathrm{cu}}^{\Sigma}}{2}
$$

To force $v_{\mathrm{cm}}$ to be exactly equal to the designed reference, $v_{\mathrm{cm}}^{\mathrm{c}}=v_{\mathrm{cm}}^{*}$ should be satisfied. Then, combining (7) and (8), the compensated terms in the $\mathrm{CM}$ voltage reference can be derived as

$$
\begin{aligned}
\Delta v_{\mathrm{cm}} & =\frac{2 v_{\mathrm{cm}}^{*} V_{\mathrm{dc}}-2 R_{\mathrm{cm}} i_{\mathrm{cm} 0} V_{\mathrm{dc}}-v_{\mathrm{s}}^{*}\left(v_{\mathrm{cl}}^{\Sigma}-v_{\mathrm{cu}}^{\Sigma}\right)}{v_{\mathrm{cu}}^{\Sigma}+v_{\mathrm{cl}}^{\Sigma}}-v_{\mathrm{cm}}^{*} \\
& \approx \frac{2 v_{\mathrm{cm}}^{*} V_{\mathrm{dc}}-v_{\mathrm{s}}^{*} v_{\mathrm{c}}^{\Delta}}{v_{\mathrm{c}}}-v_{\mathrm{cm}}^{*}
\end{aligned}
$$

where $v_{\mathrm{c}}^{\Delta}=v_{\mathrm{cl}}^{\Sigma}-v_{\mathrm{cu}}^{\Sigma}$ and $v_{\mathrm{c}}^{\Sigma}=v_{\mathrm{cu}}^{\Sigma}+v_{\mathrm{cl}}^{\Sigma}$. The arm resis-tance is generally small so it can be ignored.

\section{B. Implementation of the Compensation Algorithm}

The implementation of the compensation algorithm is depicted in Fig. 4, where the proposed CM voltage reference compensation algorithm is superimposed on the conventional 


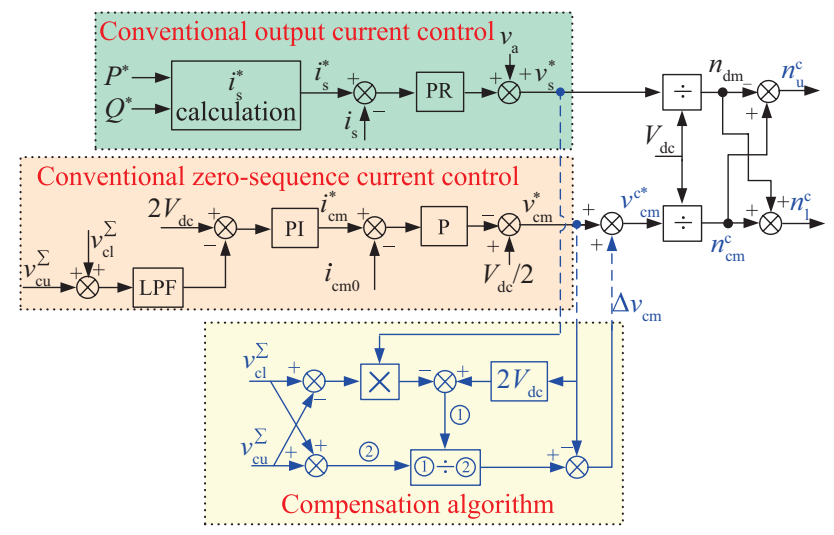

Fig. 4. The proposed compensated CCSC method for MMC.

current control strategy, which contains two control loops. One is the output current control loop with the PR regulator, whose transfer function is given as

$$
G_{\mathrm{PR}}(s)=k_{\mathrm{pr}}+\frac{k_{\mathrm{r}} s}{s^{2}+\omega_{1}^{2}}
$$

The other one is the zero-sequence current control loop used as the inner loop, the averaged sum of the two arms' capacitor voltages are controlled as the outer loop. Note that this control loop is not essential in some applications where an open loop with $v_{\mathrm{cm}}^{*}=V_{\mathrm{dc}} / 2$ is used. However, it is highly recommended that zero-sequence current should be controlled to adjust damping ratio and improve the dynamics of the system [26]. Although the averaged capacitor voltage can converge to the mean value as the direct modulation is employed [25], it can vary from $V_{\mathrm{dc}}$ under different operating condition. For example, it will increase when the MMC consumes reactive power and decrease when the MMC provides reactive power to the ac system. To avoid this problem, the averaged capacitor voltage can be controlled with a PI regulator in practice, which is the outer loop and its output is used as the zero-sequence current reference to keep the power balance. Note that the averaged sum of the two arms' capacitor voltages needs to be smoothed by a low pass filter (LPF), which avoids injecting any harmonic components into the CM current reference $i_{\mathrm{cm}}^{*}$, as shown in Fig. 4. $v_{\mathrm{cm}}^{*}$ is given as

$$
\begin{aligned}
& {v_{\mathrm{cm}}^{*}}^{*}=\frac{V_{\mathrm{dc}}}{2}-k_{\mathrm{pi}}\left(i_{\mathrm{cm}}^{*}-i_{\mathrm{cm} 0}\right) \\
& i_{\mathrm{cm}}^{*}=k_{\mathrm{pv}}\left(1+\frac{1}{\tau_{v} s}\right)\left[2 V_{\mathrm{dc}}-\operatorname{LPF}\left(v_{\mathrm{c}}^{\Sigma}\right)\right]
\end{aligned}
$$

where $k_{\mathrm{pi}}$ is the proportional gain for inner-loop current controller, $k_{\mathrm{pv}}$ and $\tau_{\mathrm{v}}$ are the proportional gain and integral time constant for the voltage controller, respectively.

Comparing to the conventional CCSC method, the frequency and sequence information of all harmonics in circulating currents are not necessary to know as there is no Park transformation or PR regulators in the compensation algorithm. The structure is very simple, as shown in Fig. 4. Moreover, the proposed compensation algorithm is designed based on the direct modulation and no additional differential energy control loop is required, which still naturally achieves the average capacitor voltage balanced of the inter-arms.

It should be noting that the calculation burden is so low that it is easy to be realized into a microprocessor. Since the essential input variables are the initial CM and DM voltage references and SM capacitor voltages, which are also measured to carry out the sorting algorithm, no additional voltage or current sensors are needed. Moreover, for both the conventional CCSC method and the proposed approach, all the capacitor voltages are detected by the local processing unit and then sent to the central ones, and then all the drive signals after closed-loop regulation are sending back to the local processing unit. As a result, the communication speed and data volume between the central processing unit and the local ones with the proposed approach are the same.

\section{Inter-Arm CApacitor Voltages Natural Balance ANALYSIS}

The voltage balancing of the inter-arm capacitors can be guaranteed by the sorting algorithm and the high switching frequency, while how the proposed CCSC technique affects the inter-arms balancing of capacitor voltages needs to be further analyzed. The overall system in Fig. 2 is time varying due to the sinusoidal components in all voltage and current variables, as well as the insertion indices, which makes complete stability analysis of the capacitor voltage difficult. However, a simple voltage balancing analysis can be obtained as the following.

First, considering that the conventional control method in Fig. 4 without the compensated algorithm is open-loop, then fixed CM and DM insertion indices $n_{\mathrm{cm}}=0.5, n_{\mathrm{dm}}=0.5 \mathrm{~m}$ $\cos \left(\omega_{1} t\right)$, which has no impact on the dynamics and thus simplify the analysis. Rearranging the equations in (3), and considering (2) and (5), which will give

$$
\begin{aligned}
& i_{\mathrm{s}}=\frac{1}{L_{\mathrm{dm}}} \int\left(\frac{n_{\mathrm{cm}} v_{\mathrm{c}}^{\Delta}+n_{\mathrm{dm}} v_{\mathrm{c}}^{\Sigma}}{2}-v_{\mathrm{a}}-R_{\mathrm{dm}} i_{\mathrm{s}}\right) \mathrm{d} t \\
& i_{\mathrm{cm}}=\frac{1}{L_{\mathrm{cm}}} \int\left(\frac{V_{\mathrm{dc}}}{2}-\frac{n_{\mathrm{cm}} v_{\mathrm{c}}^{\Sigma}+n_{\mathrm{dm}} v_{\mathrm{c}}^{\Delta}}{2}-R_{\mathrm{cm}} i_{\mathrm{cm}}\right) \mathrm{d} t
\end{aligned}
$$

In the steady state, there is no dc current in the capacitor current as the power is balanced between the dc and ac side, e.g. $i_{\mathrm{cm} 0}=1 / 4 m I_{\mathrm{s}} \cos (\varphi), n_{\mathrm{cm}} i_{\mathrm{cm} 0}$ is thus equal to $\left(1 / 2 n_{\mathrm{dm}} i_{\mathrm{s}}\right)_{\mathrm{dc}}$ in (4). Due to $i_{\mathrm{cm} 0}$ is the same in the upper and lower arms, it can thus regulate the averaged value of $v_{\mathrm{c}}^{\Sigma}$, while $i_{\mathrm{s}}$ can be used to control the averaged value of $v_{\mathrm{c}}^{\Delta}$.

Then assuming an initial inter-arm voltage unbalance occurs, e.g. the lower arm capacitor voltage $v_{\mathrm{cl}}^{\Sigma}$ steps up and becomes much more than that of the upper arm, as shown in Fig. 5(a), the averaged errors in $v_{\mathrm{c}}^{\Sigma}$ and $v_{\mathrm{c}}^{\Delta}$ can be observed. According to (12), $i_{\mathrm{s}}$ will increase and $i_{\mathrm{cm}}$ would decrease, respectively. Taking this effect back into the lower arm in (4), both of the 

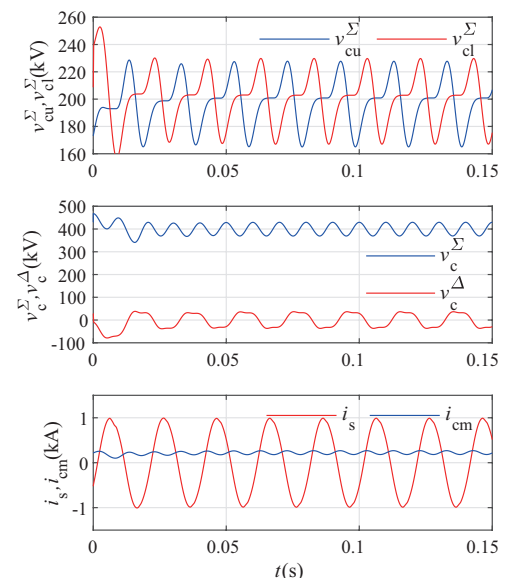

(a)
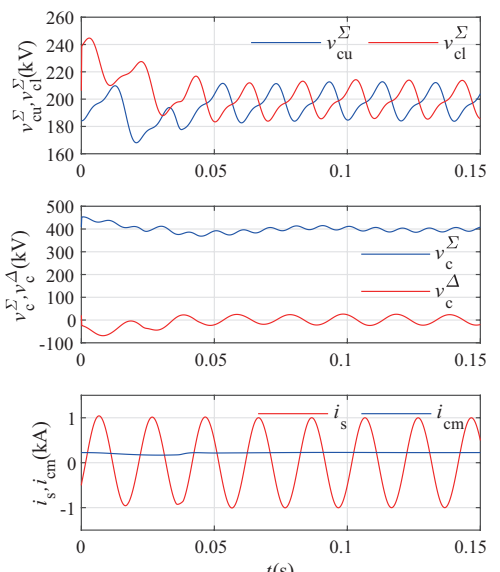

(b)
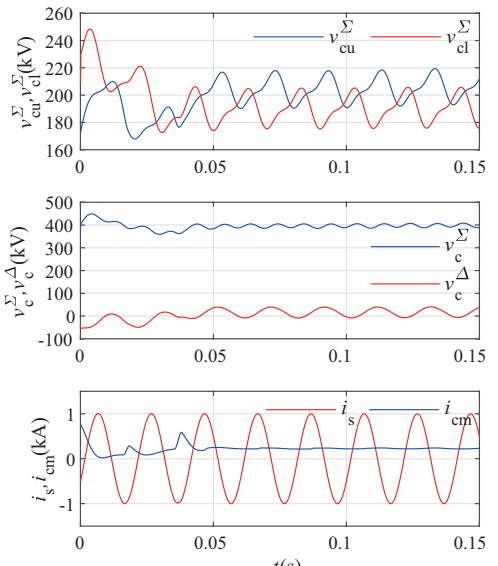

(c)

Fig. 5. Self-regulated process of the inter-arm capacitor voltage with different modulation methods. The main parameters used are in Table I in Section V, and (a) direct modulation method; (b) the proposed compensation modulation method; (c) feed-forwarding the two arms capacitor voltages as discussed in [25].

two current variations will drive $v_{\mathrm{cl}}^{\Sigma}$ back to its normal value, which is shown clearly in Fig. 5(a). This regulation procedure lasts until the averaged value of $v_{\mathrm{c}}^{\Sigma}$ finally achieves the normal value, and meanwhile the averaged $v_{\mathrm{c}}^{\Delta}$ becomes zero. A similar analysis can be realized for the upper arm capacitor voltage. Therefore, the capacitor voltages of the two arms can achieve a self-regulated balance driven by currents $i_{\mathrm{cm}}$ and $i_{\mathrm{s}}$.

Second, considering that the proposed compensation method is adopted, and all the circulating currents are suppressed well, i.e. $i_{\mathrm{cm}}$ is a purely dc current $i_{\mathrm{cm} 0}$. Substituting (8) and (9) into (12) gives

$$
\begin{aligned}
& i_{\mathrm{s}}=\frac{1}{L_{\mathrm{dm}}} \int\left(\frac{v_{\mathrm{cm}}^{*} v_{\mathrm{c}}^{\Delta}}{\Sigma}+\frac{v_{\mathrm{s}}^{*} v_{\mathrm{cu}}^{\Sigma}}{V_{\mathrm{c}}}-v_{\mathrm{a}}-R_{\mathrm{dm}} i_{\mathrm{s}}\right) \mathrm{d} t \\
& i_{\mathrm{cm}}=\frac{1}{L_{\mathrm{cm}}} \int\left(\frac{V_{\mathrm{dc}}}{2}-v_{\mathrm{cm}}^{*}-R_{\mathrm{cm}} i_{\mathrm{cm} 0}\right) \mathrm{d} t=i_{\mathrm{cm} 0}
\end{aligned}
$$

From(11) and (13), it can be seen that $i_{\mathrm{cm} 0}$ is still responsible to control the averaged value of $v_{\mathrm{c}}^{\Sigma}$ while $i_{\mathrm{s}}$ tends to regulate the averaged value of $v_{\mathrm{c}}^{\Delta}$. When $v_{\mathrm{cl}}^{\Sigma}$ increases, $i_{\mathrm{cm}}$ will decrease to regulate the averaged capacitor voltage for both arms, which can be seen clearly in Fig. 5(b), self-regulation can also be achieved in this case.

If using the closed-loop modulation with feed-forwarding the upper and lower arm capacitor voltages independently in [25], gives

$$
\begin{aligned}
& i_{\mathrm{s}}=\frac{1}{L_{\mathrm{dm}}} \int\left(v_{\mathrm{s}}^{*}-v_{\mathrm{a}}-R_{\mathrm{dm}} i_{\mathrm{s}}\right) \mathrm{d} t \\
& i_{\mathrm{cm}}=\frac{1}{L_{\mathrm{cm}}} \int\left(\frac{V_{\mathrm{dc}}}{2}-v_{\mathrm{cm}}^{*}-R_{\mathrm{cm}} i_{\mathrm{cm} 0}\right) \mathrm{d} t=i_{\mathrm{cm} 0}
\end{aligned}
$$

where it can be seen that this method can compensate both the $\mathrm{CM}$ and DM voltages efficiently and control them to the
TABLE I

System and Control Parameters Used in Simulation MMC model

\begin{tabular}{lcc}
\hline \hline Description & Symbol & Value \\
\hline AC system phase voltage & $V_{\mathrm{a}}$ & $90 \mathrm{kV}$ \\
Rated power & $S$ & $135 \mathrm{MVA}$ \\
DC voltage & $V_{\mathrm{dc}}$ & $200 \mathrm{kV}$ \\
Number of SMs per arm & $N$ & 100 \\
SM capacitance & $C$ & $4 \mathrm{mF}$ \\
Equivalent capacitor & $C / N$ & $40 \mu \mathrm{F}$ \\
SM averaged capacitor voltage & $V_{\mathrm{capmean}}$ & $1 \mathrm{kV}$ \\
Arm inductance & $L_{\mathrm{arm}}$ & $50 \mathrm{mH}$ \\
Arm resistance & $R_{\mathrm{arm}}$ & $0.3 \Omega$ \\
Proportional gain (CM current controller) & $k_{\mathrm{pi}}$ & 20 \\
Proportional gain (voltage controller) & $k_{\mathrm{pv}}$ & 1.26 \\
Integral time constant & $\tau_{\mathrm{v}}$ & 0.05 \\
Proportional gain (DM current controller) & $k_{\mathrm{pr}}$ & 200 \\
Resonant gain (DM current controller) & $k_{\mathrm{r}}$ & 31400 \\
\hline \hline
\end{tabular}

ideal values. However, it cannot contribute to the selfregulated balance as $v_{\mathrm{cu}}^{\Sigma}$ and $v_{\mathrm{cl}}^{\Sigma}$ disappear from the right of the equation in (14), which indicates $i_{\mathrm{cm}}$ and $i_{\mathrm{s}}$ are unable to regulate $v_{\mathrm{cu}}^{\Sigma}$ and $v_{\mathrm{cl}}^{\Sigma}$ Although $v_{\mathrm{c}}^{\Sigma}$ can be controlled through $i_{\mathrm{cm}}$ with an outer voltage loop as the same in Fig. 4, $v_{\mathrm{c}}^{\Delta}$ will still move from zero to a new none-zero equilibrium point as $i_{\mathrm{s}}$ cannot contribute to regulate it, as seen in Fig. 5(c). Additional energy controllers as presented in [25] are needed to achieve capacitor voltage balance between the two arms by adding extra components into $i_{\mathrm{s}}$. Overall, this method cannot achieve naturally self-regulated balance of the inter-arm capacitor voltages.

\section{Simulation Validation}

To explore the performance of the proposed CM insertion indices compensation method, a three-phase averaged simulation model of MMC is established in PSCAD/EMTDC, with the system and control parameters are listed in Table I. Note that no additional energy balance controllers have been employed in all the cases shown below. 

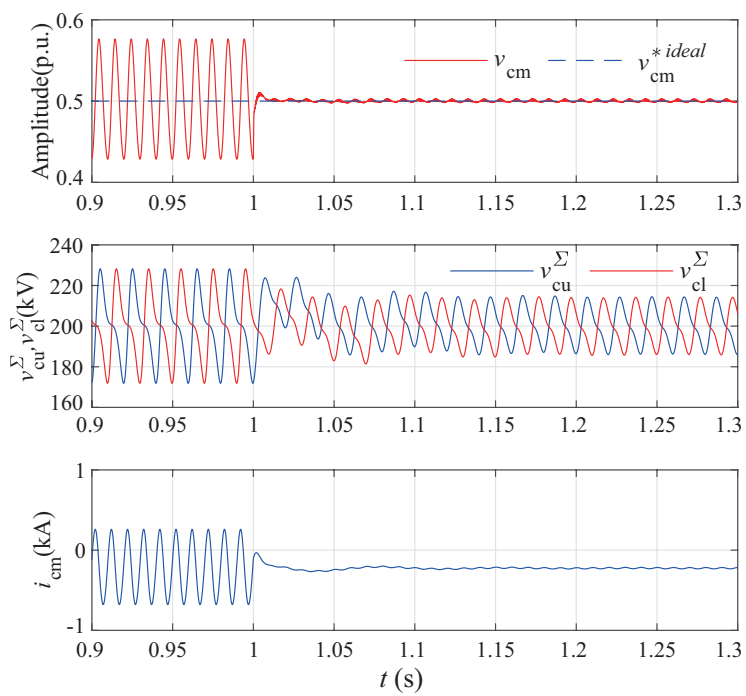

(a)



(b)

Fig. 6. Comparison of steady-state waveforms for phase A when the proposed CCSC method is enabled at $1 \mathrm{~s}$ under the operating condition $P=-1.0$ p.u., $Q=0$ p.u.

\section{A. Circulating Current Suppression Capability Under Different Operating Conditions}

Fig. 6 and 7 show the steady-state waveforms for phase A under a typical operating conditions. The proposed CCSC method is enabled at $1 \mathrm{~s}$, where the waveforms in (a) from the top to the bottom are: the desired ideal and real CM voltages, the upper and lower arm capacitor voltages and the CM current, respectively. (b) is the spectrums of $i_{\mathrm{cm}}$ for the steady states. From them, the following observations are made:

- The system can operate appropriately with the proposed compensated CCSC method.

- The circulating currents are suppressed well, which can be clearly seen from the time-domain waveforms and the spectrums of $i_{\mathrm{cm}}$.

- In contrast, there are different frequency harmonics in $i_{\mathrm{cm}}$ before $1 \mathrm{~s}$, and all of them are almost eliminated with the
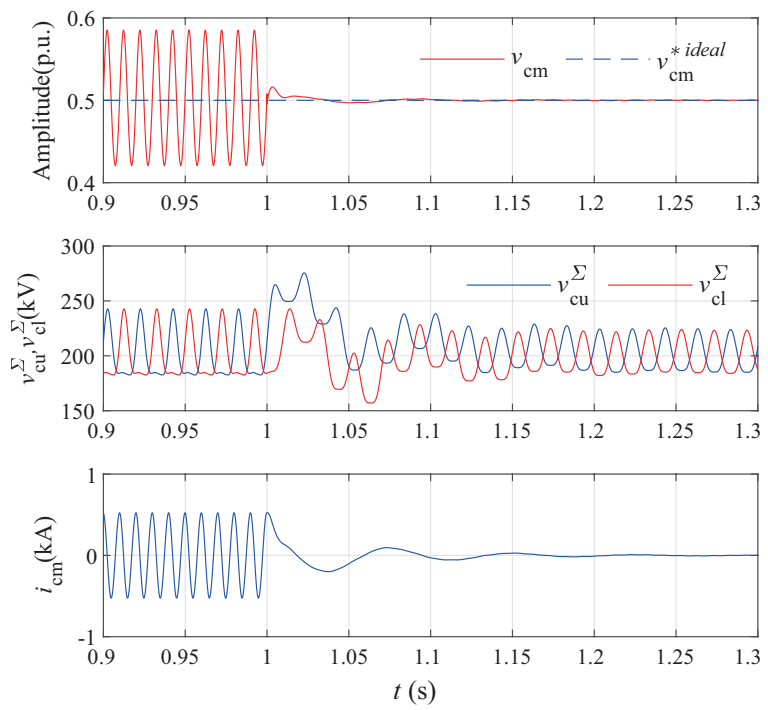

(a)



(b)

Fig. 7. Comparison of steady-state waveforms for phase A when the proposed CCSC method is enabled at $1 \mathrm{~s}$ under the operating condition $P=0$ p.u., $Q=1$ p.u..

compensation method, which can reduce the rms value of the arm current, and therefore, the efficiency of the system can finally be improved.

- Another benefit with the proposed CCSC method is that the capacitor voltage ripple is significantly reduced, which will reduce the the energy requirement and the voltage stress of insulated gate bipolar transistor (IGBT) to make the system operate more safely.

In order to extend the linear operation range and reduce the capacitor voltage ripples, a zero-sequence 3rd-order voltage harmonic can be injected into the initial DM voltage reference [18], [19], gives as

$$
v_{\mathrm{s}}^{* \prime}=v_{\mathrm{s}}^{*}-\frac{1}{6}\left|v_{\mathrm{s}}^{*}\right| \cos \left[3 \arg \left(v_{\mathrm{s}}^{*}\right)\right]
$$

here, $\left|v_{\mathrm{s}}^{*}\right|$ and $\arg \left(v_{\mathrm{s}}^{*}\right)$ are the amplitude and phase angle of $v_{\mathrm{s}}^{*}$, 

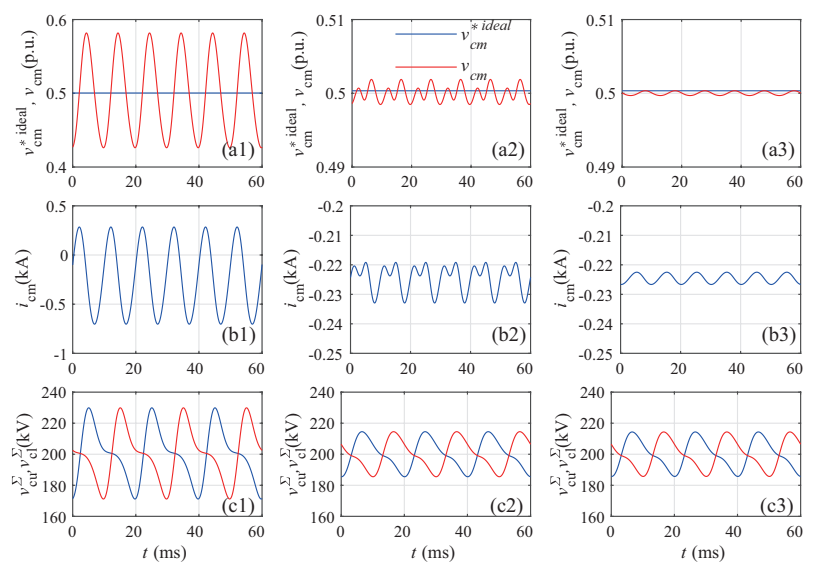

Fig. 8. Comparison of the steady-state responses with ZSHV injection for phase A with different CCSC methods under the operating condition $P=-1.0$ p.u., $Q=0$ p.u., and (a1)-(c1) without the CCSC method, (a2)-(c2) are with the conventional CCSC method using PR@2 $\omega$ regulators, (a3)-(c3) are with the proposed method.

respectively. Due to the circulating coupling effects in Fig. 3, the circulating currents will contain much more significant harmonics, especially the 2nd and 4th-order harmonics. The $\mathrm{MMC}$ with zero-sequence harmonic voltage (ZSHV) injection is also simulated to further demonstrate the effectiveness of the compensation CCSC method. Fig. 8 shows the steady-state responses for one phase with different CCSC methods. The corresponding spectrums of $i_{\mathrm{cm}}$ are depicted in Fig. 9. From these figures, in addition to the observations seen in Fig. 6, more conclusions can be obtained, given as

- The conventional CCSC method can almost eliminate the 2nd-order harmonic and can suppress part of the other harmonics in $i_{\mathrm{cm}}$ by $\mathrm{P}$ part in the PR regulators.

- Without any $\mathrm{R}$ regulators and no multi- $d q$ frame integrators, all the harmonics in $i_{\mathrm{cm}}$ can be almost eliminated by the compensation method, regardless of the frequencies and sequences information.

\section{B. Comparison of Inter-Arm Capacitor Voltages Natural Bal- ance Capability}

A test of inter-arm capacitor voltages natural balance is performed to show the advantage of the proposed approach compared to the one described in [25], which feed-forward the upper and lower arm capacitor voltages independently, as shown in Fig. 10. Before $2 \mathrm{~s}$, both the systems without CCSC method are operating in steady state and the two kinds of CCSC methods are enabled at $2 \mathrm{~s}$ for each system. As predicted from the previous analysis, when the method in [25] is used, the averaged capacitor voltages of the upper and lower arm start to diverge from each other due to the marginal stability of this strategy, as shown in Fig. 10(a). The divergence also in turn distorts $i_{\mathrm{cm}}$ from a purely dc current. In contrast, the proposed approach can maintain the capacitor voltages naturally balanced between the upper and lower arms, as shown in Fig. 10(b).

\section{Dynamic Performance Under Different Operating Condi- tions}

To further validate the effectiveness of the proposed CCSC

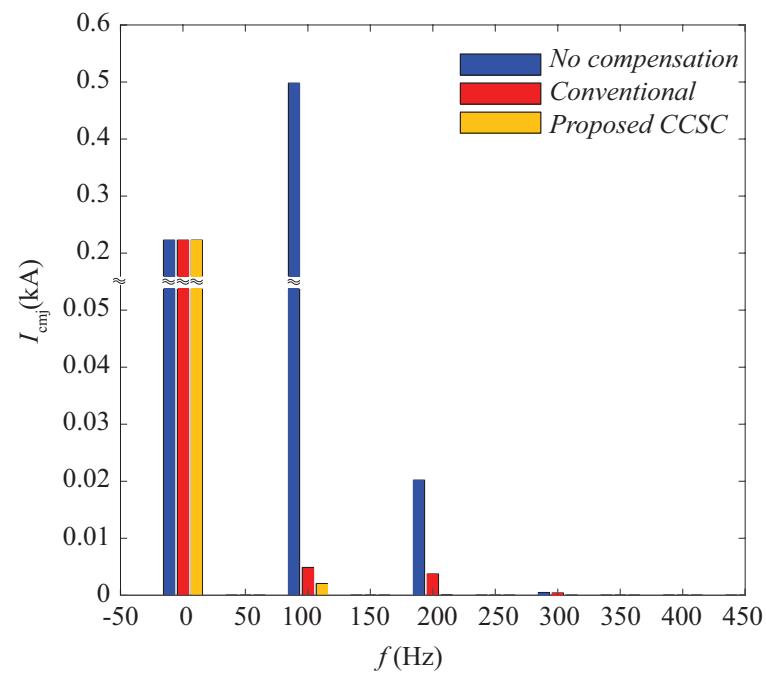

Fig. 9. The spectrums of $i_{\mathrm{cm}}$ with ZSHV injection for the steady states with different CCSC methods under the operating condition $P=-1.0, Q=0$ p.u.
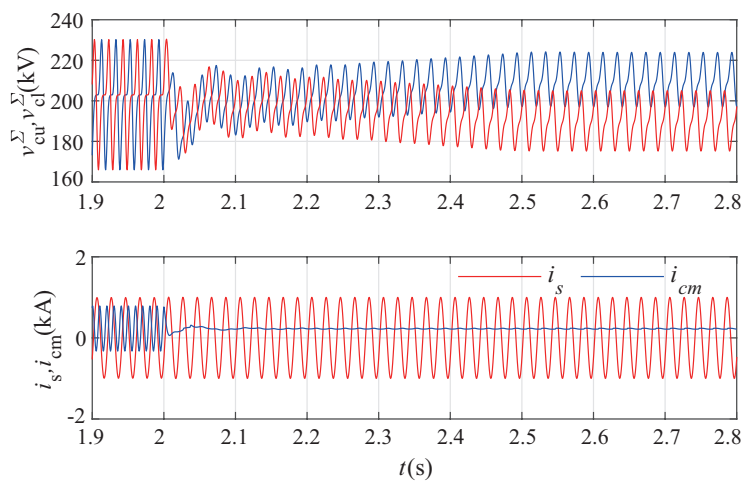

(a)
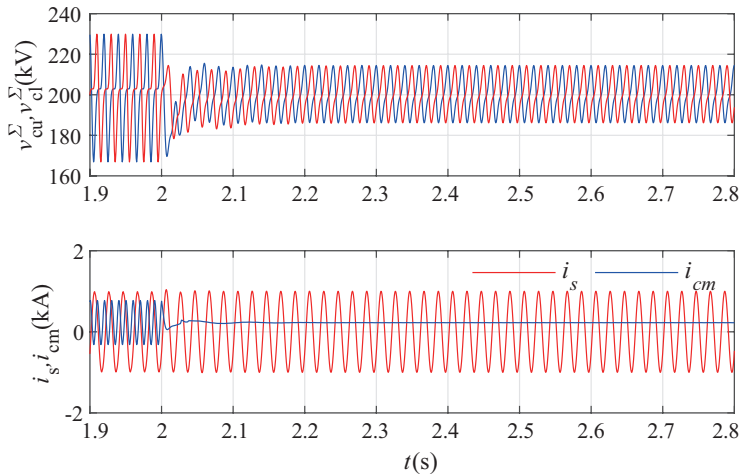

(b)

Fig. 10. The self-regulated ability of the inter-arm capacitor voltages balancing is tested under the operating condition $P=1.0$ p.u., $Q=0$ p.u. with (a) the feedforward scheme in [25], (b) the proposed CCSC method in this paper.

method, the dynamic performance of an MMC switching between different operating conditions is tested. The corresponding electrical quantities simulation results are shown in Fig. 11. Before $2 \mathrm{~s}$, the system is without CCSC method, and the rated active power is transferred from the ac grid to the dc side ( $P=-1.0$ p.u., $Q=0$ p.u.). The proposed CCSC method is enabled at $2 \mathrm{~s}$, and $0.5 \mathrm{~s}$ later, $P$ is changed to zero $(P=0$ p.u., 




(a)

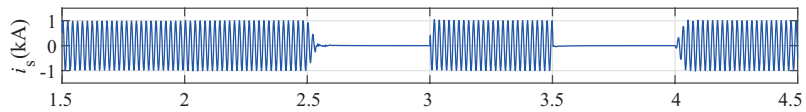

(b)

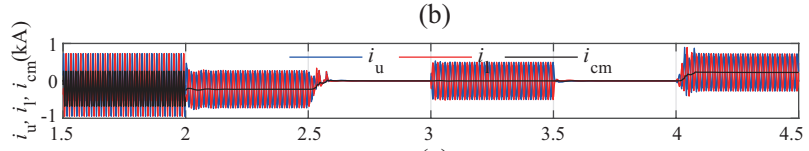

(c)



(d)

Fig. 11. Dynamic performances with the proposed compensation CCSC method.

$Q=0$ p.u.), then at $3 \mathrm{~s}, Q$ steps up to the rated reactive power ( $P=0$ p.u., $Q=1$ p.u.) and operates for $0.5 \mathrm{~s}, Q$ returns to zero again. At $4 \mathrm{~s}, \mathrm{P}$ reverses and steps from zero to the rated power ( $P=1.0$ p.u., $Q=0$ p.u.). From Fig. 11, it can be found that the system with the compensated CCSC method can accurately follow the power variation commands. The circulating currents are eliminated and $i_{\mathrm{cm}}$ becomes a pure dc current after the transient period. Moreover Fig. 11(d) also confirms again that under all conditions, the proposed method can self-regulate in order to achieve the inter-arm capacitor voltages balanced.

\section{Dynamic Performance Under Unbalanced AC Grid Conditions}

The proposed compensation approach used in a threephase MMC is also examined under an unbalanced ac grid to demonstrate the per-phase control capability of the new control scheme. As shown in Fig. 12, the grid voltage of phase A sags $20 \%$ at $2.3 \mathrm{~s}$ to simulate an unbalanced grid. From Fig. 12(b), it can be seen that the three-phase grid currents $i_{\mathrm{a}}, i_{\mathrm{b}}$ and $i_{\mathrm{c}}$ are successfully controlled to their expected references regardless of whether the ac system is balanced or not. The three phase CM currents $i_{\text {cma }}, i_{\text {cmb }}, i_{\text {cmc }}$ in Fig. 12(c) will change separately and become different under unbalanced grid conditions, which is responsible to guarantee the active power balance in each phase. Furthermore, the upper arm and lower arm capacitor voltages can also achieve naturally balanced when operate under the unbalanced ac grid, which can be clearly seen in Fig. 12(d).

\section{Conclusions}

This paper has analyzed the mechanism of the circulating currents between the phase-legs in an MMC, which revealed that due to the capacitor voltage ripples are coupled into the $\mathrm{CM}$ voltage, resulting in harmonics components that drive the circulating currents. Based on this analysis, a compensated CCSC approach is proposed by feed-forwarding the capacitor voltages to decouple the effects of the capacitor voltage ripples into the $\mathrm{CM}$ voltage. It is aimed to force the $\mathrm{CM}$ voltage

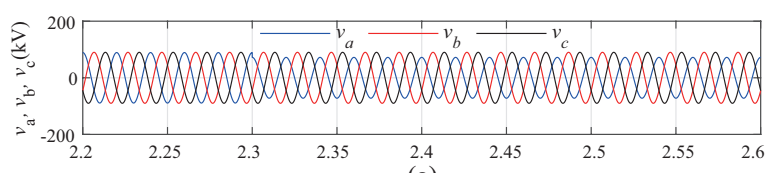

(a)



(b)

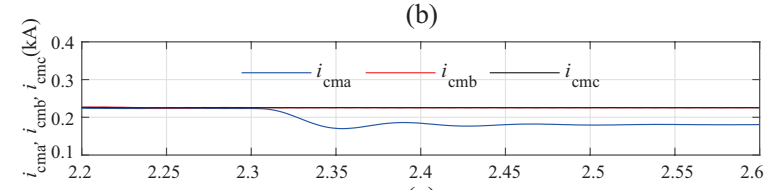

(c)

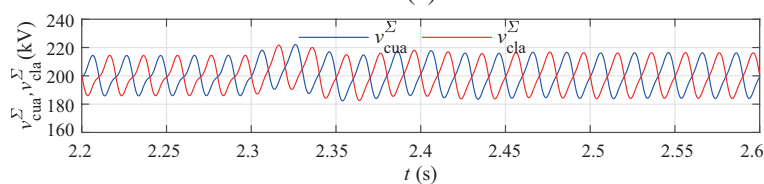

(d)

Fig. 12. Dynamic performances of with the proposed CCSC method under the unbalanced AC grid. (a) The grid voltages. (b) The grid currents (DM currents). (c) The CM currents of three phases. (d) The upper and lower arm capacitor voltages for phase A.

equal to the designed one, so that it can suppress all harmonic components in the circulating currents no matter what the harmonics frequencies and sequences are. This approach also maintains the upper and lower arm capacitor voltages naturally balanced. Compared to the conventional CCSC method with PR regulators or multi- $d q$ frame integrators, the proposed method achieves significantly a wider frequency and sequence harmonics suppression, which makes it particularly attractive for MMC applications in unbalanced, multi-frequency or variable fundamental frequency ac systems. The effectiveness of the compensation CCSC method have been verified through simulation results.

\section{REFERENCES}

[1] R. Marquart, "Stromrichterschaltungen Mit vertei-lten energiespeichern," Germany Patent DE10103031A1, Jan. 24, 2001.

[2] G. Li, D. Zhang, Y. Xin, S. Jiang, W. Wang, and J. Du, "Design of MMC hardware-in-the-loop platform and controller test scheme," in CPSS Transactions on Power Electronics and Applications, vol. 4, no. 3, pp. 143-151, Jun. 2019.

[3] A. Christe, M. Petkovic, I. Polanco, M. Utvic, and D. Dujic, "Auxiliary submodule power supply for a medium voltage modular multilevel converter," in CPSS Transactions on Power Electronics and Applications, vol. 4, no. 3, pp.204-218, Sept. 2019.

[4] A. Dekka, B. Wu, R. L. Fuentes, M. Perez, and N. R. Zargari, "Evolution of topologies, modeling, control schemes, and applications of modular multilevel converters," in IEEE Journal of Emerging and Selected Topics in Power Electronics, vol. 5, no. 4, pp. 1631-1656, Dec. 2017.

[5] Y. Okazaki, M. Hagiwara, and H. Akagi, "A speed-sensorless startup method of an induction motor driven by a modular multilevel cascade inverter," in IEEE Transactions on Industry Applications, vol. 50, no. 4, pp. 2671-2680, Jul./Aug. 2015.

[6] J. Wang, X. Han, H. Ma, and Z. Bai, "Analysis and injection control of circulating current for modular multilevel converters," in IEEE Transactions on Industrial Electronics, vol. 66, no. 3, pp. 2280-2290, Mar. 2019.

[7] B. P. McGrath, C. A. Teixeira, and D. G. Holmes, "Optimised phase 
disposition modulation of a modular multilevel converter using a state machine decoder," in Proceedings of 2015 IEEE Energy Conversion Congress and Exposition (ECCE), 2015, pp. 6368-6375.

[8] B. Li, R. Yang, D. Xu, G. Wang, W. Wang, and D. Xu, "Analysis of the phase-shifted carrier modulation for modular multilevel converters," in IEEE Transactions on Power Electronics, vol. 30, no. 7, pp. 297-310, Jul. 2015.

[9] T. Bandaru, T. Bhattacharya, and D. Chatterjee, "Modified predictive sorting algorithm for full-bridge MMC in HVDC application," in Proceedings of 2017 National Power Electronics Conference (NPEC), 2017, pp. 178-184.

[10] S. Debnath, J. Qin, B. Bahrani, M. Saeedifard, and P. Barbosa, "Operation, control, and applications of the modular multilevel converter: A review," in IEEE Transactions on Power Electronics, vol. 30, no. 1, pp. 37-53, Jan. 2015.

[11] K. Ilves, A. Antonopoulos, S. Norrga, and H. Nee, "Steady-state analysis of interaction between harmonic components of arm and line quantities of modular multilevel converters," in IEEE Transactions on Power Electronics, vol. 27, no. 1, pp. 57-68, Jan. 2012.

[12] Q. Song, W. Liu, X. Li, H. Rao, S. Xu, and L. Li, "A steady-state analysis method for a modular multilevel converters," in IEEE Transactions on Power Electronics, vol. 28, no. 8, pp. 3702-3713, Aug. 2013.

[13] B. Bahrani, S. Debnath, and M. Saeedifard, "Circulating current suppression of the modular multilevel converter in a double-frequency rotating reference frame," in IEEE Transactions on Power Electronics, vol. 31, no. 1, pp. 783-792, Jan. 2016.

[14] Q. Tu, Z. Xu, and L. Xu, "Reduced switching-frequency modulation and circulating current suppression for modular multilevel converters," in IEEE Transactions on Power Delivery, vol. 26, no. 3, pp. 2009-2017, Jul. 2011.

[15] J. Moon, C. Kim, J. Park, D. Kang, and J. Kim, "Circulating current control in MMC under the unbalanced voltage," in IEEE Transactions on Power Delivery, vol. 28, no. 3, pp. 1952-1959, Jul. 2013.

[16] Y. Zhou, D. Jiang, J. Guo, P. Hu, and Y. Liang, "Analysis and control of modular multilevel converters under unbalanced conditions," in IEEE Transactions on Power Delivery, vol. 28, no. 4, pp. 1986-1995, Oct. 2013.

[17] M. Vasiladiotis, N. Cherix, and A. Rufer, "Accurate capacitor voltage ripple estimation and current control considerations for grid-connected modular multilevel converters," in IEEE Transactions on Power Electronics, vol. 29, no. 9, pp. 4568-4579, Sep. 2014.

[18] K. Ilves, S. Norrga, L. Harnefors, and H. P. Nee, "On energy storage requirements in modular multilevel converters," in IEEE Transactions on Power Electronics, vol. 29, no. 1, pp. 77-88, Jan. 2014.

[19] C. Zhao, Z. Wang, Z. Li, P. Wang, and Y. Li, "Characteristics analysis of capacitor voltage ripples and dimensioning of full-bridge MMC with zero sequence voltage injection," in IEEE Journal of Emerging and Selected Topics in Power Electronics, vol. 7, no. 3, pp. 2106-2115, Sep. 2019.

[20] X. She, A. Huang, X. Ni, and R. Burgos, "AC circulating currents suppression in modular multilevel converter," in Proceedings of IECON 2012 - 38th Annual Conference on IEEE Industrial Electronics Society, Montreal, QC, Canada, 2012, pp. 191-196.

[21] Z. Li, P. Wang, Z. Chu, H. Zhu, Y. Luo, and Y. Li, "An inner current suppressing method for modular multilevel converters," in IEEE Transactions on Power Electronics, vol. 28, no. 11, pp. 4873-4879, Nov. 2013.

[22] S. Li, X.Wang, Z.Yao, T. Li, and Z. Peng, "Circulating current suppressing strategy for MMC-HVDC based on nonideal proportional resonant controllers under unbalanced grid conditions," in IEEE Transactions on Power Electronics, vol. 30, no. 1, pp. 387-397, Jan. 2015.

[23] L. B. Brahim, A. Gastli, M. Trabelsi, K. A. Ghazi, M. Houchati, and H. A. Rub, "Modular multilevel converter circulating current reduction using model predictive control," in IEEE Transactions on Industrial Electronics, vol. 63, no. 6, pp. 3857-3866, Jun. 2016.

[24] L. He, K. Zhang, J. Xiong, and S. Fan, "A repetitive control scheme for harmonic suppression of circulating current in modular multilevel converters," in IEEE Transactions on Power Electronics, vol. 30, no. 1, pp. 471-481, Jan. 2015.

[25] A. Antonopoulos, L. Ängquist, and H. Nee, "On dynamics and voltage control of the modular multilevel converter," in Proceedings of 13th European Conference on Electronics and Applications, 2009, pp. 1-10.
[26] L. Harnefors, A. Antonopoulos, S. Norrga, L. Ängquist, and H. Nee, "Dynamic analysis of modular multilevel converters," in IEEE Transactions on Industrial Electronics, vol. 60, no. 7, pp. 2526-2537, Jun. 2013.

[27] L. Ängquist, A. Antonopoulos, D. Siemaszko, K. Ilves, M. Vasiladiotis, and H. Nee, "Open-loop control of modular multilevel converters using estimation of stored energy," in IEEE Transactions on Industry Applications, vol. 47, no. 6, pp. 2516-2524, Nov./Dec. 2011.

[28] A. Antonopoulos, L. Ängquist, L. Harnefors, K. Ilves, and H. Nee, "Global asymptotic stability of modular multilevel converters," in IEEE Transactions on Industrial Electronics, vol. 61, no. 2, pp. 603-612, Feb. 2014.

[29] L. Harnefors, A. Antonopoulos, K. Ilves, and H. Nee, "Global asymptotic stability of current-controlled modular multilevel converters," in IEEE Transactions on Power Electronics, vol. 30, no. 1, pp. 249-258, Jan. 2015.

[30] Y. Sun, C. A. Teixeira, D. G. Holmes, B. P. McGrath, and J. Zhao, "Low order circulating current suppression of PWM-based modular multilevel converters using dc-link voltage compensation," in IEEE Transactions on Power Electronics, vol. 33, no. 1, pp. 210-224, Jan. 2018.

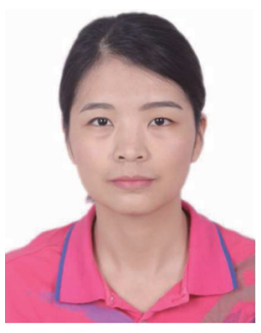

Xiaoling Xiong received the B.Eng., M.Eng. and $\mathrm{Ph} . \mathrm{D}$. degrees in electrical engineering from Nanjing University of Aeronautics and Astronautics, Nanjing, China, in 2007, 2010 and 2015 respectively. She had worked as a Research Assistant in Department of Electronic and Information Engineering at Hong Kong Polytechnic University from February, 2011 to July, 2012.

Since 2015, she has been with North China Electric Power University, Beijing, where she was a lecturer. Simultaneously, she has been with Aalborg University, Aalborg, Denmark from December 2018, where she was a visiting Post-Doctoral with the Department of Energy Technology. Her current research interests include HVDC system, modeling, analysis and design power electronic systems and study the nonlinear behaviors in power electronic circuits.



Xiongfei Wang received the B.S. degree from Yanshan University, Qinhuangdao, China, in 2006, the M.S. degree from Harbin Institute of Technology, Harbin, China, in 2008, both in electrical engineering, and the Ph.D. degree in energy technology from Aalborg University, Aalborg, Denmark, in 2013.

Since 2009, he has been with the Department of Energy Technology, Aalborg University, where he became an Assistant Professor in 2014, an Associate Professor in 2016, a Professor and Research Program Leader for Electronic Power Grid (eGrid) in 2018, and the Director of Aalborg University-Huawei Energy Innovation Center in 2020. His current research interests include modeling and control of grid-interactive power converters, stability and power quality of converter-based power systems, active and passive filters.

Dr. Wang was selected into Aalborg University Strategic Talent Management Program in 2016. He has received six IEEE Prize Paper Awards, the 2016 Outstanding Reviewer Award of IEEE TRANSACTIONS ON POWER ELECTRONICS, the 2018 IEEE PELS Richard M. Bass Outstanding Young Power Electronics Engineer Award, the 2019 IEEE PELS Sustainable Energy Systems Technical Achievement Award, and the 2019 Highly Cited Researcher by Clarivate Analytics (former Thomson Reuters). He serves as a Member at Large for Administrative Committee of IEEE Power Electronics Society in 2020-2022, and as an Associate Editor for the IEEE Transactions on Power Electronics, the IEEE Transactions on Industry Applications, and the IEEE Journal of Emerging and Selected Topics in Power Electronics. 


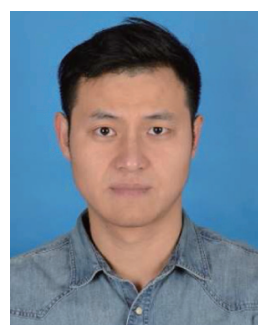

Dong Liu received the B.Sc. and M.Sc. degree in electrical engineering from the Nanjing University of Science and Technology in China, in 2003 and 2007, respectively, received Ph.D. from China Electric Power Research Institute in 2012; from 2018 to 2019, he was a Post-doc in Aalborg University in Denmark. His current research interests include power electronic control, stability and interaction in voltage source converter and MMC based DC transmission system, and physical and digital simulation technology.



Frede Blaabjerg was with ABB-Scandia, Randers, Denmark, from 1987 to 1988. From 1988 to 1992, he got the Ph.D. degree in Electrical Engineering at Aalborg University in 1995. He became an Assistant Professor in 1992, an Associate Professor in 1996, and a Full Professor of power electronics and drives in 1998. From 2017 he became a Villum Investigator. $\mathrm{He}$ is honoris causa at University Politehnica Timisoara (UPT), Romania and Tallinn Technical University (TTU) in Estonia.

His current research interests include power electronics and its applications such as in wind turbines, PV systems, reliability, harmonics and adjustable speed drives. He has published more than 600 journal papers in the fields of power electronics and its applications. He is the co-author of four monographs and editor of ten books in power electronics and its applications.

He has received 32 IEEE Prize Paper Awards, the IEEE PELS Distinguished Service Award in 2009, the EPE-PEMC Council Award in 2010, the IEEE William E. Newell Power Electronics Award 2014, the Villum Kann Rasmussen Research Award 2014, the Global Energy Prize in 2019 and the 2020 IEEE Edison Medal. He was the Editor-in-Chief of the IEEE Transactions on Power Electronics from 2006 to 2012. He has been Distinguished Lecturer for the IEEE Power Electronics Society from 2005 to 2007 and for the IEEE Industry Applications Society from 2010 to 2011 as well as 2017 to 2018. In 2019-2020 he serves a President of IEEE Power Electronics Society. He is Vice-President of the Danish Academy of Technical Sciences too. He is nominated in 2014-2019 by Thomson Reuters to be between the most 250 cited researchers in Engineering in the world.

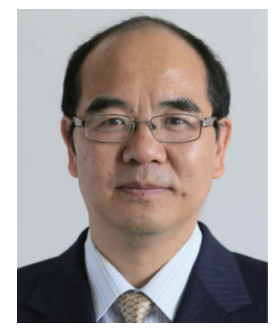

Chengyong Zhao received the Ph.D. degree in electrical engineering from North China Electric Power University, Beijing, China, in 2001. He became an teacher and currently a full professor of electrical engineering with North China Electric Power University, Beijing, China. His current research interests include high volatge direct current (HVDC) transmisson systems and power electronicbased power system. 